\title{
Energy localization, Fano resonances, and nonlinear meta-optics
}

\author{
Yuri Kivshar \\ Nonlinear Physics Center, Australian National University, Canberra ACT 2601, Australia \\ ITMO University, St. Petersburg 197101, Russia \\ E-mail: ysk@internode.on.net
}

Received January 22, 2018, published online May 28, 2018

\begin{abstract}
This paper reflects on some memories of the research topics developed at Department No. 29 of the Institute for Low Temperature Physics and Engineering in Kharkov more than 30 years ago. It also provides some recent advances on my major research activities related to those topics, including energy localization and solitons in nonlinear lattices, Fano resonances in photonics and phononics, and nonlinear effects in meta-optics and nanophotonics. Curiously enough, each of those topics can be associated with some memories and discussions that happened in Kharkov long time ago.

PACS: $45.05 .+\mathrm{x}$ General theory of classical mechanics of discrete systems;

63.20.Pw Localized modes;

42.25.Hz Interference;

42.65.Ky Frequency conversion; harmonic generation, including higher-order harmonic generation.
\end{abstract}

Keywords: localized modes, metamaterials, Fano resonances, nanophotonics, nonlinear meta-optics.

\section{Introduction}

Research topics at Department No. 29 of the Institute for Low Temperature Physics and Engineering in Kharkov were quite diverse, but they were mainly based on ideas from solid states physics and theory of magnetism, in spite of the inherent connection of Prof. Arnold Kosevich to the foundations of electromagnetic theory (James Maxwell), wave scattering (Lord Rayleigh) and backward electromagnetic waves (Arthur Schuster), as shown in Fig. 1.

The study of nonlinear localized modes was one of the major research directions in the department of Arnold Kosevich. This topic was initiated by the pioneering paper [1] that introduced a new type of self-localized states in a chain with nonlinear interactions or/and on-site potentials, later rediscovered by A. Sievers and S. Takeno [2] as "intrinsic localized mode”. Such modes appear in homogeneous anharmonic chains of identical particles that support strongly localized oscillatory modes with the frequencies above the upper edge of the phonon spectrum band. The existence of such modes is due to strong nonlinear interaction and weak coupling between particles, and such modes are also known to appear in different settings [3,4]; these days they are termed also as "discrete breathers" [5] being associated with solitons in discrete systems [6].
Another broad area of research was associated with the study of localized solutions (domain walls, solitons, vortices) of the semiclassical Landau-Lifshitz equations describing the nonlinear spin dynamics in magnetism. This background environment and discussions were probably responsible for my later interest in metamaterials. As a matter of fact, after attending the Winter School in Kaurovka, my senior colleague Dr. Alexander Kovalev mentioned about a strange talk of Victor Veselago who

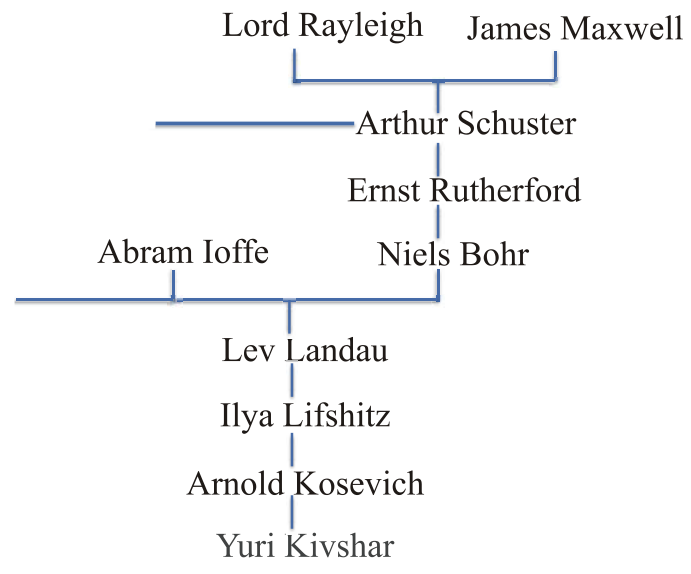

Fig. 1. Academic supervisor tree for Arnold Kosevich as obtained with the help of the search engine, https://academictree.org/physics/. 
presented a hypothetical material with negative refraction. Much later, such properties were realized in electromagnetic metamaterials, artificial media in which subwavelength electromagnetic constituents replace atoms as the basic structural elements to control the light-matter interaction, and they led to the prediction and observation of many novel electromagnetic phenomena that are not available or much weaker in natural materials [7-11]. This includes negative index of refraction, invisibility cloaking, giant chirality, etc.

Many of such important phenomena are the manifestation of optical magnetism, predicted and observed in specifically designed artificial subwavelength structures that allow strong magnetic response, even when such structures are made of non-magnetic materials [12]. Indeed, although real quantumorigin magnetism in its conventional sense is not available at high optical frequencies, the metamaterial concept allows to design artificial "meta-atoms" to engineer macroscopic magnetic permeability $\mu$ and magnetic response by achieving strong resonances in structured non-magnetic systems, through the spatial dispersion and nonlocal electric-field effects, inducing a strong magnetic dipole moment.

\section{Energy localization}

Recent studies revealed different scenarios of the energy localization and the formation of high-frequency localized modes, solely driven by deformations of the chains and formation of the regions with a stress gradient. In deformed lattices, there appear regions with stronger coupling that support high-frequency localized states. This scenario of the energy localization can be realized only in the chains with anharmonic interatomic interactions whereas any deformation of linear arras with local harmonic interactions between the neighboring particles would not lead to any energy localization.

The importance of curved surfaces in localization has been already revealed in other fields. In particular, it was shown that a curved graphene surface can play a role of an effective potential for phonons supporting spatially localized phonons [13]. Similarly, graphene plasmons can be localized by effective geometric potentials [14].

To illustrate the basic physics of the deformation-induced energy localization, we follow the recent original paper [15] and consider a linear chain of particles with the near-neighbor anharmonic interactions. We assume, without restriction of generality, that all particles have identical masses (normalized as $m=1$ ) being placed in a chain with the lattice spacing $a=1$ $\left(V(a)=0, V^{\prime}(a)=0\right)$, and the interaction is characterized by the stiffness coefficient $\kappa=V^{\prime \prime}(a)=1$. We describe the interaction between neighboring particles by the well-known Lennard-Jones atomic potential

$$
V(r)=\frac{1}{72}\left(r^{-6}-1\right)^{2}=4 \varepsilon\left[\left(\frac{\sigma}{r}\right)^{12}-\left(\frac{\sigma}{r}\right)^{6}\right]+\varepsilon,
$$

where energy $\varepsilon=1 / 72$, parameter $\sigma=2^{-1 / 6}$, and the external interaction is introduced by the linear potential $U(x)=-F x$, that corresponds to the action of a constant force $F$ on all particles (an analogue of the gravity force or constant electric field).

The action of a constant force leads to inhomogeneous deformations of the couplings of particles in the chain, with a linear gradient of tension. The strongest deformation is experienced by the fixed edge of the chain that "holds" the entire structure. Due to anharmonic coupling between particles, the bond at the left edge become "softer" for $F>0$. Therefore, the energy can be localized at the right end of the chain for $F>0$ [see Fig. 2(a)] in the form of high-frequency oscillatory states.

We notice that the formation of localized states in inhomogeneously deformed chain is a direct consequence of the anharmonic interatomic interaction between the particles in the chain. Under the action of the constant force, the harmonic chain is stretched inhomogeneously with the formation of a linear gradient of deformations, but all coupling coefficients will have the same stiffness. Such undeformed chain will not support the existence of localized modes.

Modeling of the thermal conductivity reveals [15] that the external force induces an effective anisotropy in the chain for $F>0$. A heat transfer against the action of the

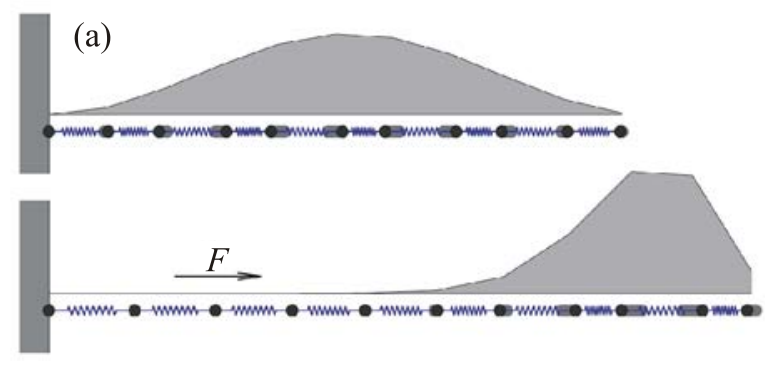

(b)

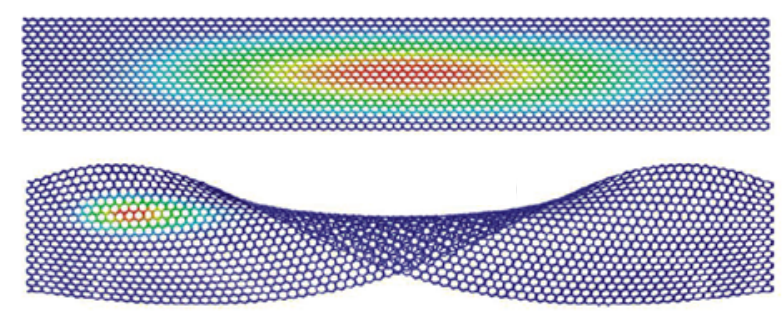

Fig. 2. (Color online) (a) Schematic presentation of the LennardJones chain of particles with a fixed left end for (above) no force and (below) stretched chain under the action of the force $F$. Shading show the high-frequency oscillations in the chain. Curve above the chain corresponds to energy distribution of highfrequency eigenmode of the chain [15]. (b) Spatial distribution of the energy of localized oscillations in a nanoribbon of the size $19.53 \times 4.11 \mathrm{~nm}$, for flat and twisted nanoribbons (the twisting angle is $\phi=270^{\circ}$ ) [15]. 
force becomes more preferential. The anisotropy grows with an increase of the applied force, and the thermal flow reduces [15]. This is explained by the fact that for larger values of the force smaller number of particles interact with the thermostat at the left edge. The effect of anisotropy depends on the temperature difference $\Delta T=T_{+}-T_{-}$, so that anisotropy vanishes for $\Delta T \rightarrow 0$.

For two-dimensional systems, strong deformations can be realized by twisting or bending the structure. As an example, we mention a nanoribbon cut off a graphene sheet with the zigzag edges. The study of twisted nanoribbons has been conducted in several papers [16-18] with the main focus on stability due to severe twists and nonelastic deformations. Here consider the case when all bonds remain but become stretched due to weak twists.

We consider a planar nanoribbon and fix the positions of the edge atoms with the first indices $n=1$ and $n=N_{X} / 2$. Then, we twist the nanoribbon along the length by the angle $\phi$ and find the positions of atoms in a new equilibrium state $\left\{u_{n, k}^{0}\right\}_{n=1, k=1}^{N, K}$ corresponding to the twisted geometry shown in Fig. 2(b). For such a twisting, the strongest deformed area appear along the edges of the nanoribbon where the valent bonds are stretched, whereas the bonds in the center remain weakly deformed. As a result, we observe the formation of localized states at the middle of the nanoribbon [15].

Thus, recent studies revealed that inhomogeneous deformations (stretching, compression, twisting, or bending) of anharmonic lattices can lead to a local change of the coupling coefficients and induce the energy localization of high-frequency phonon modes. For a linear chain of particles interacting via the Lennard-Jones potentials under the action of a constant external force, we have demonstrated [15] that high-frequency oscillations can be localized at the edge of the inhomogeneously deformed chain. Additionally, this mechanism is responsible for the formation of spatially localized phonon states in twisted graphene nanoribbons and the topological Möbius-like graphene structures through stretching of the valent bonds between carbon atoms [15]. These novel anharmonic effects can be employed for rectification and control of heat flows in stretched lattices at the nanoscale.

\section{Fano resonances}

First, I heard about the Fano resonance after one of the seminars of Department No. 29 when Arnold Kosevich, while commenting on some results presented at the seminar, sketched a peak on a growing density of states and on a blackboard and said that the discussed resonant transmission can be associated with the so-called "Fano resonance". This was also related to the topic of one of his later papers [19] that, as he said, was based on very old results obtained during his work with Academician Ilya Lifshitz.
Much later, this concept re-appeared in connection with our studies of defect and waveguides in photonic crystals where Fano resonances appear very naturally being associated with the resonant transmission of light.

Indeed, nanophotonics is dealing with extremely strong and confined optical fields at subwavelength scales far beyond the diffraction limit. A proper combination of photonic nanoparticles can support Fano resonances arising from interference between different modes and a continuum of radiative electromagnetic waves. Fano resonance is a fascinating phenomenon of the wave physics observed across various branches of optics, including photonics, plasmonics, and metamaterials. In nanophotonic structures, the Fano resonances manifest themselves as resonant suppression of the total scattering cross-section accompanied by an enhanced absorption. Fano resonances are able to confine light more efficiently, and they are characterized by a steeper dispersion than conventional resonances, which make them promising for nanoscale biochemical sensing, switching or lasing applications.

It is commonly believed that Fano resonances in nanophotonics originate solely from the excitation of plasmonic modes being associated with plasmonic hot spots. In a sharp contrast to this common belief, recently we demonstrated experimentally [21] that subwavelength nanoparticles with high refractive index can support strong Fano resonances through interference of optically-induced magnetic rather than electric dipole resonant modes, as was predicted theoretically [20] and later observed experimentally [21].

One of the nanophotonic structures we studied experimentally is shown in Fig. 3(a). From the general intuition, we expect that a narrow resonant mode should be associated with the central nanoparticle, whereas other six nanoparticles of different radii form a broad supermode. The interference of these two collective modes leads to the suppression of the total scattering associated with the Fano resonances [20].

However, our rigorous analysis [22] revealed principal differences between dielectric and plasmonic oligomers. First, in comparison with plasmonic oligomers, all-dielectric structures are less sensitive to the separation between the particles, since the field is localized inside dielectric nanoparticles. Second, dielectric nanostructures exhibit almost negligible losses which allow observing the Fano resonances in new geometry not available for plasmonic counterparts. These novel features lead to different coupling mechanisms between nanoparticles making all-dielectric oligomer structures very attractive for various applications in nanophotonics.

Linear optical transmittance spectra of four silicon nanodisk heptamers with varying central nanodisk diameter are measured with unpolarized incident light, and the results are shown in Fig. 3(b). Fano resonance can be spectrally tuned by a size variation of the central particle, due to linear dependence of magnetic dipole resonance on the diameter [23]. Magnetic Fano resonances are less sensitive 
(a)
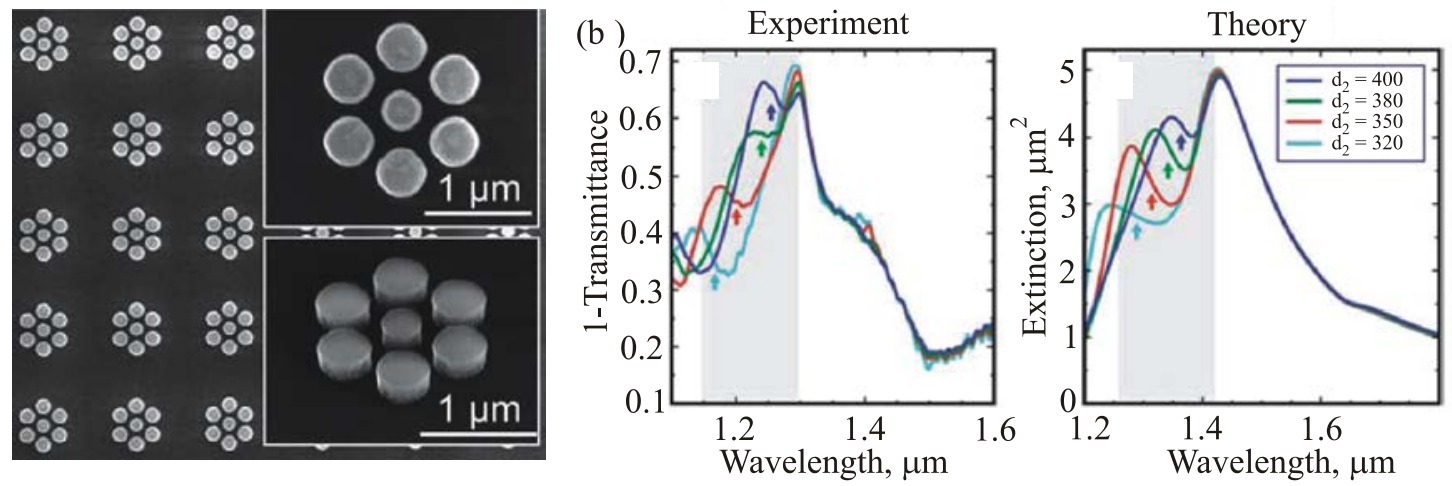

(c)

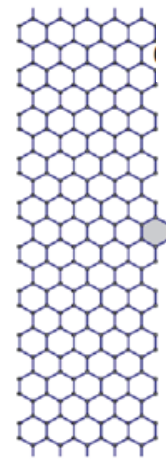

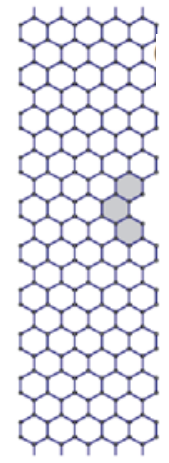

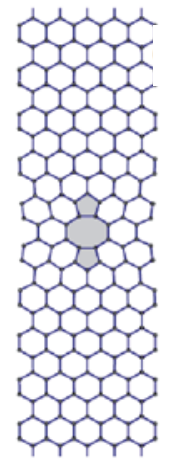

(d)

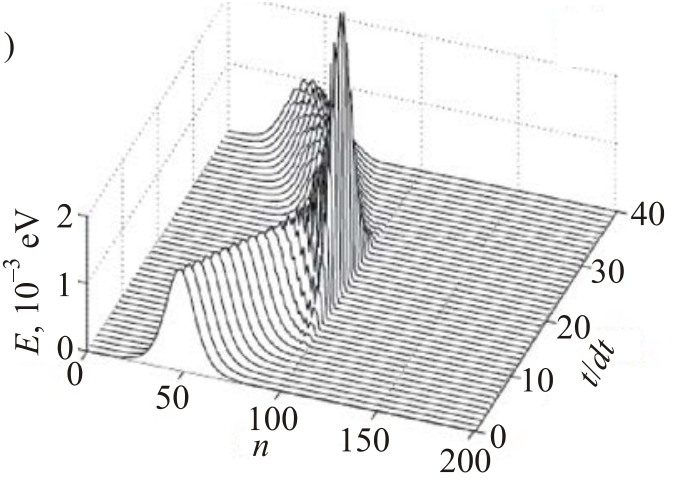

Fig. 3. (Color online) (a), (b) Photonic Fano resonances in optical nanoparticle oligomers composed of silicon nanodisks. Shown in (b) are experimentally measured and theoretically calculated extinction spectra for a variety of silicon nanodisk heptamer structures featuring a systematic variation of the central nanodisk diameter. Fano resonance is observed as a minimum that moves across the spectrum when the diameter of the central nanoparticle is varied [21]. (c) Localized defects in armchair graphene nanoribbon that show the manifestation of phononic Fano resonance [24]. (d) Fano-induced reflection of a wavepacket of the in-plane phonons in a nanoribbon with the double vacancy $V_{2}(585)$. Shown is the energy distribution at different time values $t$ along the nanoribbon ( $n$ is the number of the elementary unit cell, time step is $d t=0.15 \mathrm{ps}$ ).

to unintentional structural variations like surface roughness, which makes them very promising for many applications in nanophotonics. Moreover, owing to their higher resistance to heat as compared to optical metal nanostructures and to a possibility of free carrier generation in the material, magnetic Fano resonances might offer a range of new opportunities not attainable with plasmonic structures, for example harshenvironment sensing or nonlinear applications.

A very interesting effects of phononic Fano resonances have been observed for the interaction between localized vibrational modes and propagating phonons in graphene nanoribbons with different types of localized internal and edge defects [24], and it is directly related to the simplified model considered by A. Kosevich [19]. Savin and Kivshar analyzed discrete eigenmodes of the nanoribbons with defects [as shown in Fig. 3(c)] and observed a partial suppression of the phonon transport due to the phononic Fano resonances originating from interference between localized and propagating phonons, reveled through reflection of phonon wavepackets, see Fig. 3(d). The Fano resonance is also manifested in the reduction of the heat transport along the graphene stripe, when each of the local defects reduces the amount of the heat flow transmitted through the nanoribbon, with the effect being more pronounced at low temperatures when the thermal energy transfer is dominated by the phonon transport.

Thus, rapid progress in photonics and nanotechnology brings many examples of resonant optical phenomena associated with the physics of Fano resonances, with many applications in sensing, optical switching, and pulse shaping. For a successful design of photonic devices, it is crucially important to have a deep understanding of different resonant phenomena. Last year, we reviewed a broad range of resonant electromagnetic effects using a model of two coupled oscillators including the Fano resonance and electromagnetically induced transparency, Kerker and Borrmann effects, as well as parity-time symmetry breaking [25]. We have discussed how to introduce the Fano parameter that describes a transition between two fundamental but seemingly different spectroscopic signatures associated with the asymmetric Fano and symmetric Lorentzian shapes. In addition, we have discussed the recent advances in the physics of Fano resonances in resonant nanoparticle arrays and dielectric metasurfaces. 


\section{Nonlinear meta-optics}

The recent developments of the physics of high-index dielectric nanoparticles [26-29] suggests the importance of an alternative mechanism of light localization in subwavelength optical structures via low-order dipole and multipole Mie resonances [30] that may generate magnetic response via the displacement current contribution. According to the Mie theory, dielectric nanoparticles can exhibit strong magnetic resonances in the visible. A magnetic resonance originates from the excitation of a particular electromagnetic mode inside the nanoparticle with a circular displacement current of the electric field. This mode is excited when the wavelength of light is comparable to the particles diameter. It has an antiparallel polarization of the electric field at the opposite sides of the particle while the magnetic field is oscillating up and down at the middle. The study of Mie-resonant dielectric nanostructures has been established as a new research direction in modern nanoscale optics and metamaterial-inspired nanophotonics [26-29]. Because of their unique optically induced electric and magnetic geometry-driven resonances, high-index nanophotonic structures are expected to complement or even replace different plasmonic components in a range of potential applications.

Second-harmonic generation (SHG) is one of the most important nonlinear processes in optics. In SHG, the frequency of an incident light beam is doubled inside of a nonlinear crystal. SHG is nowadays employed in many applications, including laser sources and nonlinear microscopy. The simplest example is a green laser pointer employing an efficient frequency-doubling nonlinear crystal and emitting light at $532 \mathrm{~nm}$.

Usually, nonlinear optics relies on bulk nonlinear crystals, such as lithium niobate. Unfortunately, these materials are difficult to integrate with other devices, due to the difficulties inherent in their manufacturing and machining, and they are also not cost-effective. Furthermore, special phase-matching conditions are often required in order to obtain useful conversion efficiencies. Although the output beam profile in bulk crystals can be engineered by complex periodic poling, this technique is not easily accessible due to its requirement for a spatially inhomogeneous distribution of high voltages across the crystals.

To overcome these issues, it would be useful if we could replace bulk nonlinear crystals with ultrathin surfaces composed of nanocrystals that can generate SHG with high efficiency. Such nonlinear meta-atoms could also be used to manipulate the SHG radiation to form complex beams with arbitrary patterns.

Recent progress of meta-optics suggest that AlGaAs nanocrystals fabricated on a glass substrate not only enable a nonlinear conversion efficiency exceeding $10^{-4}$ (for subwavelength-thick structures), but also provide an opportunity to define the beam shape and polarization in both forward and backward directions [31]. The generation of complex and high-efficiency vector beams offers unique opportunities for many novel applications such as nonlinear microscopy, nonlinear holography, sum-frequency and difference-frequency generation, spontaneous parametric down-conversion, and parametric amplification. A wide range of opportunities requires much deeper studies of nonlinear optics at the nanoscale.

Within the macroscopic description based on Maxwell's equations, the light-matter interaction is described in the electric dipole approximation specified by the nonlinear relationship between the applied electric field $\mathbf{E}$ and induced polarization $\mathbf{P}$ as (for nonmagnetic media)

$$
\mathbf{P}=\varepsilon_{0}\left[\ddot{\chi}^{(1)} \cdot \mathbf{E}+\ddot{\chi}^{(2)}: \mathbf{E E}+\ddot{\chi}^{(3)}: \mathbf{E E E}+\ldots\right],
$$

written as a Taylor series in E. In this approach, the first term describes the linear regime at weak external fields, and $\vec{\chi}^{(N)}$ are the $N$ th order susceptibility tensors of the rank $N+1$, which describe the polarization dependent parametric interaction and symmetries of the specific nonlinear media. Usually, optical nonlinearities of natural materials are weak, and the nonlinear effects manifest themselves for strong applied electromagnetic fields, generated by powerful and coherent sources of light. Because considerable amounts of the electromagnetic energy can be confined to tiny volumes in nanoparticles or even smaller hot spots, they enable downscaling the required optical powers, because the intensity of parametric processes scales with the fourth or sixth power of the fundamental field strength.

Under high intensities of light, an anharmonic motion of electrons produces photons at frequencies different from that of the incoming light, with SHG and third-harmonic generation (THG) as prominent examples. It is a widely adopted concept that only the electric polarization of the material is affected by the anharmonic motion of the electrons driven by the electromagnetic field. However, metamaterials can exhibit nonlinear magnetic response resulting in a new type of magnetic nonlinearities [32]. This picture is at large adapted in the microwave regime, and a vast number of research groups are engaged in utilizing tailored nonlinear response of metamaterials, as discussed in the recent review papers [33,34]. However, the idea of generic magnetic nonlinearities remains distant to the nonlinear optics community, while new direct observations of the effects induced by magnetic response are being reported, as discussed below.

An important issue in nonlinear optics is the crystalline symmetry. In particular, within the electric dipole approximation of the light-matter interaction, the second-order nonlinear effects are not possible in uniform centrosymmetric media such as plasmonic metals and group IV semiconductors, because of the simple symmetry constraints, while no such restriction exists for the third-order 
nonlinear processes [35]. However, the inversion symmetry can be broken at surfaces and interfaces, thus enabling the second-order nonlinear processes to occur being generated from surfaces of isotropic media due to the electric-dipole surface contribution to the effective nonlinear polarization. Sensitivity of such nonlinear effects to the properties of surfaces can be used in probing and sensing techniques. Importantly, the bulk nonlinear polarization originates from higher-order nonlocal magnetic-dipole and electric-quadrupole interactions with light at the microscopic level. To account for such multipolar effects, we can present the effective light-matter interaction Hamiltonian in the form [36,37]

$$
H_{\text {int }}=-\mathbf{p} \cdot \mathbf{E}-\mathbf{m} \cdot \mathbf{B}-[\mathbf{Q} \nabla] \cdot \mathbf{E}-\ldots,
$$

where $\mathbf{E}$ is a local electric field, $\mathbf{p}$ is the electric dipole moment, $\mathbf{m}$ is the magnetic dipole moment, and $\mathbf{Q}$ is the electric quadrupole moments.

Strong interaction of subwavelength structures with an external light field may occur due to the resonant excitation of low-order modes (up to quadrupoles) due to surface plasmon resonances (in plasmonic materials, such as metals and doped graphene), or Mie-type resonances (in dielectric nanoparticles with high refractive index) being supported by large displacement currents. The physics of these resonances can be understood from the classical problem of the Mie scattering by spherical particles. The macroscopic source of nonlinear polarization is defined by the induced near-field distribution, and it is expressed in terms of multipolar bulk and surface contributions inherent to the nonlinear medium. Nonlinear response functions appear due to averaging multipolar light-matter interactions, which can be described by the Hamiltonian $H_{\text {int }}$ at the microscopic level, with account for symmetry and structural properties of the material. The induced nonlinear polarization generate electric and magnetic multipoles of different orders, yielding nontrivial radiation and polarization patterns through the far-field interference. In the framework of this approach, the "optical magnetic" Mie mode can support strong fields that drive the electric-dipoleallowed bulk nonlinearity of the material, and in turn, the nonlinear source induced in the nanoparticle can give rise to higher-order multipoles in nonlinear scattering.

In the last few years, the role of nonlinear magnetic response in optics was intensively addressed. Shcherbakov et al. [38] employed a novel type of optically magnetic nanostructures to show that they can significantly enhance nonlinear conversion, as summarized in several images of Figs. 4(a)-(d). The laser beam is focused on a nanoparticle [see Fig. 4(a)], and excite the internal resonances. A silicon nanoparticle supports the Mie-type modes including the fundamental electric and magnetic dipolar mode [Figs. 4(b), (c)]. Along with being optically magnetic, they do not suffer from intrinsic losses in the infrared and are CMOScompatible. Shcherbakov et al. [38] confirmed that the
THG response from silicon nanodisks prevails over the THG from the bulk silicon by using THG microscopy and THG spectroscopy techniques. The low intrinsic losses of the disks made it possible to reach conversion efficiencies high enough for the generated UV light to be observed even under the table-lamp illumination [see Fig. 4(d)]. Bringing the nanodisks in the oligomer arrangement provides another degree of freedom for nonlinear magnetoelectric coupling [39], see Fig. 4(e).

Similar studies of the multipolar enhancement of SHG have been conducted for hybrid metal-dielectric nanoparticles [40]. In order to straightforwardly identify the magnetic dipolar contribution to optical nonlinearities, the authors performed spectrally resolved second-harmonic measurements and multipolar analysis of the SHG from hybrid nanodisk. The latter can sustain anti-symmetric movement of electron plasma leading to the resonant magnetic response. They employed the multipolar decomposition of the experimental and calculated SHG spectra, and observed that the main contribution to the detected nonlinear signal originates from the conventionally neglected electric quadrupolar and magnetic dipolar sources [40].

Generation of different localized modes can reshape completely the physics of nonlinear effects at the nanoscale [41]. Utilizing the Mie resonances in resonant dielectric nanoparticles has recently been recognized as a promising strategy to gain higher efficiencies of nonlinear parametric processes at low modal volumes, and novel functionalities originating from optically-induced magnetic response. In particular, it is possible not only discover novel regimes of nonlinear optical magnetism in nanoantennas [42], but also to distinguish experimentally nonlinear contributions of electric and magnetic responses by analysing the structure of polarization states of vector beams in SHG with continuously tuning polarization of the optical pump [42]. Generated electric multipoles can be distinguished from magnetic multipoles in the far-field region by their distinct polarization. For example, an electric dipole polarization follows the polar angle unit vector, whereas in the field generated from a magnetic dipole mode follows the azimuthal angle unit vector. This results in the radial polarization from an electric dipole in the projection plane perpendicular to the dipole moment and, correspondingly, azimuthal polarization distribution from a magnetic dipole [see Fig. 4(f)].

Another important mechanism for enhancing nonlinear interactions at the nanoscale is to employ the multipolar interference for creating a tight field confinement in the socalled optical anapole mode [43,44]. This anapole mode is associated with the concept of nonradiating current configurations, and in optics it can be viewed as an engineered superposition of electric and toroidal optical dipole moments resulting in destructive interference of the radiation fields. This may happen due to similarity of the far-field scattering patterns generated by an optical dipole mode and toroidal modes, and usually for the generation of toroidal modes in metamaterials some more complex structures of 

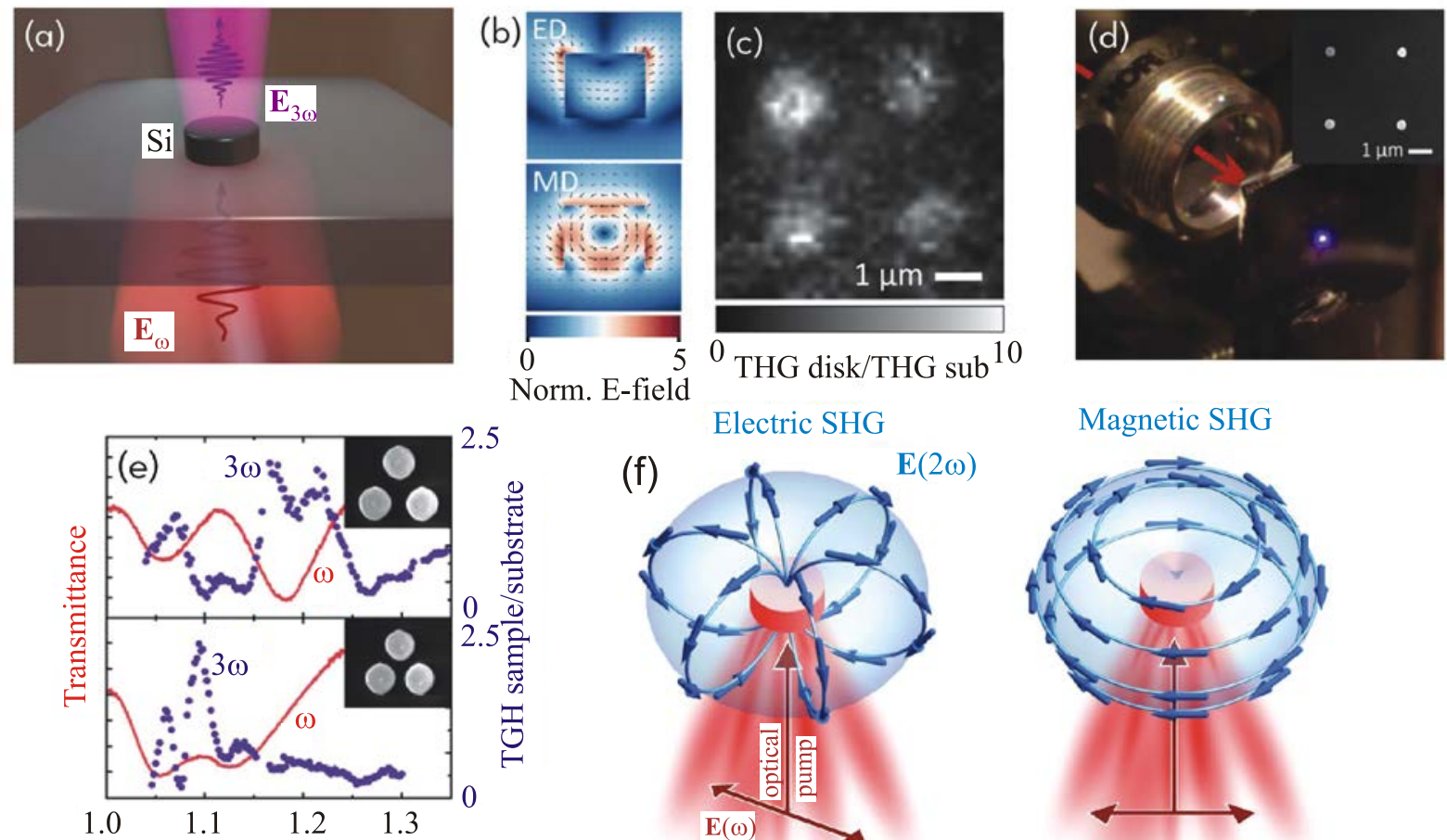

Norm. E-field
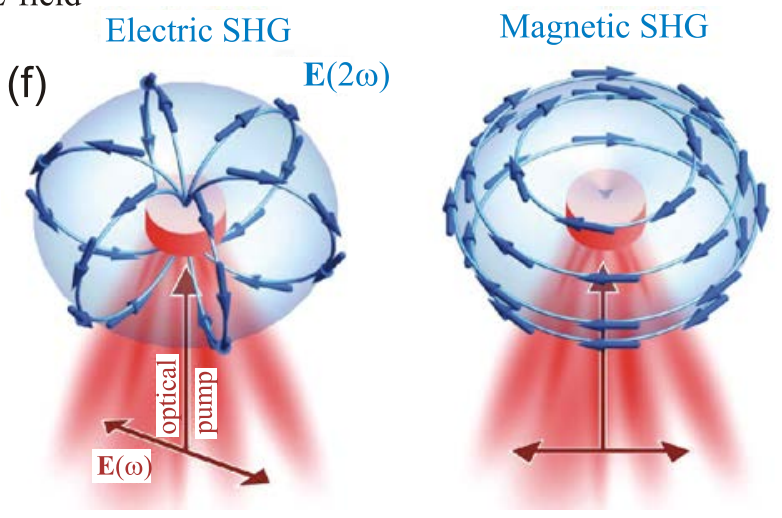

Fig. 4. (Color online) Nonlinear harmonic generation at the nanoscale. (a)-(d) THG from a Si dielectric nanoparticle with the magnetic Mie resonance. Local fields calculated for the electric dipolar and magnetic dipolar modes, respectively. Map of the THG intensity from the nanodisks normalized over the THG from a bulk silicon slab. Eye-visible THG from the silicon nanodisk array [38]. (e) Coupling of nanodisks within oligomers tailors the nonlinear-optical response [39]. (f) Electric vs. magnetic SHG in nanoantennas, with distinct polarization patterns for the generated electric and magnetic multipoles [42].

nanoparticles are suggested [45]. However, dielectric nanoparticles can support the (almost radiationless) anapole mode allowing an overlap of the toroidal and electric dipole modes through a geometry tuning, with a pronounced dip in the far-field scattering accompanied by the specific near-field distribution [43]. Importantly, these anapole modes can be employed to resolve a major challenge with the efficient coupling of light to nanoscale optical structures. Indeed, as was shown theoretically [44], a nanoscale laser based on a tightly confined anapole mode in InGaAs nanodisks allows the efficient coupling of light into waveguide channels with four orders of magnitude intensity than classical nanolasers, as well as the generation of ultrafast (of $100 \mathrm{fs}$ ) pulses via spontaneous mode locking of several anapoles, offering an attractive platform for integrated photonics sources for advanced and efficient nanoscale circuitry.

Recently, Grinblat et al. [46,47] employed the property of the anapole mode for the observation of strong thirdorder nonlinear processes and efficient THG from Ge nanodisks. They observed a pronounced valley in the scattering cross section at the anapole mode, while the electric field energy inside the disk is maximized due to high confinement within the dielectric nanoparticle. Grinblat $e t$ al.
[46] investigated the dependence of the third harmonic signal on the size of a nanodisk and pump wavelength and revealed the main features of the anapole mode, corresponding to an associated THG conversion efficiency of $0.0001 \%$ at an excitation wavelength of $1650 \mathrm{~nm}$, which is four orders of magnitude greater than the case of an unstructured germanium reference film. Furthermore, they concluded that the nonlinear conversion via the anapole mode outperforms that via the radiative dipolar resonances by about at least one order of magnitude.

The most recent developments in the study of the optical response of dielectric nanostructures with high refractive index is associated with the high values of the quality $(Q)$ factor of the resonances due to bound states in the continuum [48] and supercavity modes [49]. Indeed, it was recently shown that strong coupling between eigenmodes can be observed in a single subwavelength high-index dielectric resonator, being associated with the mode transformation and Fano resonances revealed for varying resonators aspect ratio. For such bound states in the continuum the radiative losses are almost suppressed due to the destructive interference, and this can lead to the values of the $Q$-factor about 200, as shown recently [48]. 


\section{Concluding remarks}

Many discussions of various aspects of nonlinear physics and magnetism during my stay in Kharkov laid the foundation of many research activities I have been undertaken since that time. Traditionally, this involved the problems of energy localization in nonlinear arrays, later evolving into the study of optical solitons in waveguide arrays and nonlinear optical lattices. Historical links of Arnold Kosevich to James Maxwell revealed through the analysis of the academic tree of Fig. 1, eventually pushed my research towards optics and nanophotonics where old ideas were found to be useful, from Fano resonances to artificial magnetic response in optics. The latest activity in alldielectric meta-optics moves towards nonlinear and active structures and practical metadevices, defined as the devices having unique and useful functionalities that are realized by structuring of functional matter on the subwavelength scale. Meta-optics will ultimately employ all of the advantages of optically-induced magnetic resonances and shape many important applications, including optical sensing, parametric amplification, fast spatial modulation of light for telecom applications, nonlinear active media, as well as both integrated classical and quantum circuitry and topological photonics, underpinning a new generation of nanoantennas, nanolasers, highly-efficient metasurfaces, and ultrafast metadevices.

\section{Acknowledgments}

I thank many of my colleagues and collaborators involved into the research activities briefly summarized above, especially K. Chong, A. Fedyanin, S. Kruk, A. Savin, and M. Shcherbakov. I thank A. Kovalev for the invitation to contribute to this special issue and his critical reading of the manuscript.

1. A.M. Kosevich and A.S. Kovalev, J. Exp. Theor. Phys. 40, 891 (1975).

2. A.J. Sievers and S. Takeno, Phys. Rev. Lett. 61, 970 (1988).

3. A.S. Dolgov, Sov. Phys. Solid State 28, 907 (1986).

4. J.B. Page, Phys. Rev. B 41, 7835 (1990).

5. S. Flach and C.R. Willis, Phys. Rep. 295, 181 (1998).

6. Yuri S. Kivshar and Govind P. Agrawal, Optical Solitons: From Fibers to Photonics Crystals, Academic Press (2003).

7. D.R. Smith, J.B. Pendry, and M.C.K. Wiltshire, Science 305, 788 (2004).

8. V.M. Shalaev, Nature Photonics 1, 41 (2007).

9. C.M. Soukoulis and M. Wegener, Nature Photonics 5, 523 (2011).

10. Y. Liu and X. Zhang, Chem. Soc. Rev. 40, 2494 (2011).

11. N.I. Zheludev and Y.S. Kivshar, Nat. Mater. 11, 917 (2012).

12. Y.S. Kivshar and A.P. Roberts, Fiz. Nizk. Temp. 43, 1119 (2017) [Low Temp. Phys. 43, 895 (2017)].

13. A.V. Savin and Y.S. Kivshar, Appl. Phys. Lett. 94, 111903 (2009).
14. D. Smirnova, S.H. Mousavi, Z. Wang, Y.S. Kivshar, and A.B. Khanikaev, ACS Photonics 3, 875 (2016).

15. A.V. Savin and Yu.S. Kivshar, Phys. Rev. B 96, 064307 (2017).

16. Y. Li, J. Phys. D: Appl. Phys. 43, 495405 (2010).

17. A.S. Kochnev and I.A. Ovid'ko, Rev. Adv. Mater. Sci. 43, 77 (2015).

18. D. Xia, Q. Li, Q. Xue, C. Liang, and M. Dong, Phys. Chem. Chem. Phys. 18, 18406 (2016).

19. A.M. Kosevich, J. Exp. Teor. Phys. 88, 168 (1999).

20. A.E. Miroshnichenko and Y.S. Kivshar, Nano Lett. 12, 6459 (2012).

21. K.E. Chong, B. Hopkins, I. Staude, A.E. Miroshnichenko, J. Dominguez, M. Decker, D.N. Neshev, I. Brener, and Y.S. Kivshar, Small 10, 1985 (2014).

22. B. Hopkins, A.N. Poddubny, A.E. Miroshnichenko, and Y.S. Kivshar, Phys. Rev. A 88, 053819 (2013).

23. I. Staude, A.E. Miroshnichenko, M. Decker, N.T. Fofang, S. Liu, E. Gonzales, J. Dominguez, T.S. Luk, D.N. Neshev, I. Brener, and Y. Kivshar, ACS Nano 7, 7824 (2013).

24. A.V. Savin and Y.S. Kivshar, Sci. Rep. 7, 4668 (2017).

25. M.F. Limonov, M.V. Rybin, A.N. Poddubny, and Y.S. Kivshar, Nature Photonics 11, 543 (2017).

26. A.I. Kuznetsov, A.E. Miroshnichenko, M.L. Brongersma, Y.S. Kivshar, and B. Luk’yanchuk, Science 354, aag2472 (2016).

27. S. Kruk and Y.S. Kivshar, ACS Photonics 4, 2638 (2017).

28. I. Staude and J. Schilling, Nature Photonics 11, 274 (2017).

29. Z.J. Yang, R. Jiang, X. Zhuo, Y.-M. Xie, J. Wang, and H.-Q. Liu, Phys. Rep. 701, 1 (2017).

30. G. Mie, Annalen der Physik 330, 377 (1908).

31. D. Neshev, R. Camacho-Morales, M. Rahmani, S. Kruk, L. $\mathrm{Xu}$, D. Smirnova, A. Solntsev, A. Miroshnichenko, H.H. Tan, F. Karouta, S. Naureen, K. Vora, L. Carletti, C. De Angelis, C. Jagadish, and Y.S. Kivshar, SPIE Newsroom.

32. A. Rose, D. Huang, and D.R. Smith, Phys. Rev. Lett. 110, 063901 (2013).

33. M. Lapine, I. Shadrivov, and Y. Kivshar, Rev. Mod. Phys. 86, 1093 (2014).

34. M. Lapine, Phys. Status Solidi B 254, 1600452 (2017).

35. M. Lippitz, M. A. van Dijk, and M. Orrit, Nano Lett. 5, 799 (2005).

36. L. Novotny and B. Hecht, Principles of Nano-Optics, Cambridge University Press, New York (2007).

37. M. Kasperczyk, S. Person, D. Ananias, L.D. Carlos, and L. Novotny, Phys. Rev. Lett. 114, 163903 (2015).

38. M.R. Shcherbakov, D.N. Neshev, B. Hopkins, A.S. Shorokhov, I. Staude, E.V. Melik-Gaykazyan, M. Decker, A.A. Ezhov, A.E. Miroshnichenko, I. Brener, A.A. Fedyanin, and Y.S. Kivshar, Nano Lett. 14, 6488 (2014).

39. M.R. Shcherbakov, A.S. Shorokhov, D.N. Neshev, B. Hopkins, I. Staude, E.V. Melik-Gaykazyan, A.A. Ezhov, A.E. Miroshnichenko, I. Brener, A.A. Fedyanin, and Y.S. Kivshar, ACS Photonics 2, 578 (2015). 
40. S.S. Kruk, M. Weismann, A.Y. Bykov, E.A. Mamonov, I.A. Kolmychek, T. Murzina, N.C. Panoiu, D.N. Neshev, and Y.S. Kivshar, ACS Photonics 2, 1007 (2015).

41. D. Smirnova and Y.S. Kivshar, Optica 3, 1241 (2016).

42. S. Kruk, R. Camacho-Morales, L. Xu, M. Rahmani, D.A. Smirnova, L. Wang, H.H. Tan, C. Jagadish, D.N. Neshev, and Y.S. Kivshar, Nano Lett. 17, 3914 (2017).

43. A.E. Miroshnichenko, A.B. Evlyukhin, Y.F. Yu, R.M. Bakker, A. Chipouline, A.I. Kuznetsov, B. Luk'anchuk, B.N. Chichkov, and Y.S. Kivshar, Nat. Commun. 6, 8069 (2015).

44. J.S. Totero Gongora, A.E. Miroshnichenko, Y.S. Kivshar, and A. Fratalocchi, Nat. Commun. 8, 15535 (2017).
45. T. Kaelberer, V.A. Fedotov, N. Papasimakis, D.P. Tsai, and N.I. Zheludev, Science 330, 1510 (2010).

46. G. Grinblat, Y. Li, M.P. Nielsen, R.F. Oulton, and S.A. Maier, Nano Lett. 16, 4635 (2016).

47. G. Grinblat, Y. Li, M.P. Nielsen, R.F. Oulton, and S.A. Maier, ACS Nano 11, 953 (2017).

48. M.V. Rybin, K.I. Koshelev, Z.F. Sadrieva, K.B. Samusev, A.A. Bogdanov, M.F. Limonov, and Y.S. Kivshar, Phys. Rev. Lett. 119, 243901 (2017).

49. M. Rybin and Y. Kivshar, Nature (London) 541, 164 (2017). 